\title{
Differential Mechanisms of the Effect of Peroxisome Proliferator-Activated Receptor Gamma Agonists on Bleomycin-Induced Lung Fibrosis
}

\author{
Keisuke Miyamoto ${ }^{1}$, Sadatomo Tasaka $^{1 *}$, Yasushi Nakano ${ }^{1}$, \\ Hiromi Shinoda ${ }^{1}$, Hirofumi Kamata ${ }^{1}$, Wakako Yamasawa ${ }^{2}$, \\ Makoto Ishii $^{1}$, Naoki Hasegawa ${ }^{3}$, Tomoko Betsuyaku ${ }^{1}$ \\ ${ }^{1}$ Division of Pulmonary Medicine, Keio University School of Medicine, Tokyo, Japan \\ ${ }^{2}$ Department of Laboratory Medicine, Keio University School of Medicine, Tokyo, Japan \\ ${ }^{3}$ Center for Infectious Diseases and Infection Control, Keio University School of Medicine, Tokyo, Japan \\ Email: "tasaka@cpnet.med.keio.ac.jp
}

Received March 1, 2013; revised April 3, 2013; accepted April 11, 2013

Copyright (C) 2013 Keisuke Miyamoto et al. This is an open access article distributed under the Creative Commons Attribution License, which permits unrestricted use, distribution, and reproduction in any medium, provided the original work is properly cited.

\begin{abstract}
Background and Objectives: Peroxisome proliferator-activated receptor- $\gamma($ PPAR- $\gamma)$ is a nuclear receptor whose activation regulates inflammation and fibrosis in various organs. We aimed to investigate the effect of two PPAR- $\gamma$ ligands, telmisartan and rosiglitazone, on lung injury and fibrosis induced by intratracheal bleomycin (BLM). Methods: Lung injury and fibrosis was induced in female $\mathrm{C} 57 \mathrm{Bl} / 6$ mice by intratracheal instillation of $1.0 \mathrm{mg} / \mathrm{kg}$ of BLM. Some of the animals received rosiglitazone intraperitoneally or telmisartan in drinking water. Bronchoalveolar lavage (BAL) was performed 2, 7, 14 or 21 days after BLM instillation for cell counting and measurement of mediators in the lung. In a separate series, the lungs were sampled for collagen assay and histopathological evaluation. Results: Treatment with rosiglitazone or telmisartan significantly attenuated the BLM-induced increases in lung collagen content, pathological score, and inflammatory cells in BAL fluid. Rosiglitazone significantly suppressed BLM-induced elevation of TGF- $\beta_{1}$, MCP-1, and IL-6 levels in the lung. In contrast, telmisartan made no changes in these cytokines, whereas it mitigated the BLM-induced increase in prostaglandin $\mathrm{F}_{2 \alpha}$ in the lung. Higher concentrations of rosiglitazone and telmisartan attenuated proliferation of lung fibroblasts in vitro. Conclusions: Two PPAR- $\gamma$ ligands, rosiglitazone and telmisartan, exert protective effects on BLM-induced lung fibrosis through the suppression of different profibrotic mediators.
\end{abstract}

Keywords: Peroxisome Proliferator-Activated Receptor- $\gamma$, Bleomycin; Pulmonary Fibrosis; Transforming Growth Factor- $\beta_{1}$; Prostaglandin $\mathrm{F}_{2 \alpha}$

\section{Introduction}

Idiopathic pulmonary fibrosis is a progressive, lifethreatening, interstitial lung disease that is characterized morphologically by thickening of the alveolar septa with collagen deposition and myofibroblast proliferation, and by a diffuse inflammatory infiltrate [1]. Much of the information regarding the development of pulmonary fibrosis has been acquired with a well-characterized animal model in which lung fibrosis is induced by intratracheal administration of the antineoplastic agent bleomycin (BLM) [2]. In rodents, BLM administration induces acute inflammatory response followed by fibroblast proliferation and increased collagen content in the lung [3]. "Corresponding author.
Various mediators are involved in the pathogenesis of pulmonary fibrosis, although the detailed mechanisms are still not well understood $[4,5]$.

Peroxisome proliferator-activated receptor- $\gamma(\mathrm{PPAR}-\gamma)$, which is a member of a family of ligand-activated nuclear transcriptional factors, plays a critical role in normal lung development, injury, and repair [6,7]. PPAR- $\gamma$ ligands inhibit a variety of inflammatory actions in macrophages, including expression of inducible nitric oxide synthase, superoxide dismutase, gelatinase, matrix metalloproteinase and several interleukins $[8,9]$. There is accumulating evidence indicating that PPAR- $\gamma$ is an important regulator of inflammation, fibrosis and immune responses in various diseases including pancreatic fibrosis, liver fibrosis and collagen vascular diseases [10]. 
Rosiglitazone (RGZ), a member of thiazolidinedione family of antidiabetic agents, binds PPAR- $\gamma$ with high affinity [11]. Genovese and colleagues showed that RGZ reduces BLM-induced lung injury in mice [12]. The activation of PPAR- $\gamma$ by RGZ reduces inflammatory cell infiltration and the expression of inducible nitric oxide synthase [12]. Telmisartan (TS), an angiotensin receptor blocker (ARB), has been shown to bind PPAR- $\gamma$ and exert an agonistic effect $[13,14]$. Otsuka and coworkers reported that candesartan cilexetil, another ARB, ameliorated morphological changes and an increased amount of hydroxyproline in lung homogenates induced by intratracheal BLM [15]. However, the effect of TS on the BLM-induced lung fibrosis has not been evaluated. In addition, the detailed mechanisms of the antifibrotic effect of PPAR- $\gamma$ ligands remain to be determined.

In the present study, we evaluated the inflammatory response to intratracheal BLM and subsequent fibrotic changes in the lung, comparing the BLM-control mice without administration of a PPAR- $\gamma$ agonist and those treated with TS or RGZ. To determine the effect of PPAR- $\gamma$ agonists on the accumulation of inflammatory cells, we collected BAL fluid 2, 7, 14, or 21 days after the BLM challenge. The mice that received instillation of sterile phosphate-buffered saline (PBS) served as controls. The development of lung fibrosis was evaluated with lung pathology and measurement of the collagen content in the lung. The levels of transforming growth factor- $\beta_{1}$ (TGF- $\left.\beta_{1}\right)$, monocyte chemotactic peptide-1/CC chemokine ligand 2 (MCP-1/CCL2), and interleukin-6 (IL-6), which are known to be involved in the pathogenesis of lung fibrosis, and prostaglandin $\mathrm{F}_{2 \alpha}\left(\mathrm{PGF}_{2 \alpha}\right)$, a lipid mediator that is associated with fibrosis, were also measured in BAL fluid [16-20]. In addition, an MTT assay was performed to examine the effect of PPAR- $\gamma$ agonists on the proliferation of mouse lung fibroblasts (MLFs).

\section{Methods}

\subsection{Animals}

Female C57/B16 mice were purchased from CLEA Japan (Tokyo, Japan) and routinely bred in the vivarium of Keio University School of Medicine, Tokyo, Japan. All experiments were performed with mice at the age of 8 12 weeks. All animal experiments were approved by the Animal Care and Use Committee of Keio University School of Medicine.

\subsection{Model of Intratracheal Bleomycin Injury}

Mice (20 - $25 \mathrm{~g}$ ) were anesthetized with intraperitoneal ketamine $(120 \mathrm{mg} / \mathrm{kg})$ and xylazine $(12 \mathrm{mg} / \mathrm{kg})$. Intratracheal instillation of BLM $(1 \mathrm{mg} / \mathrm{kg})$ or PBS in a volume of $2 \mathrm{~mL} / \mathrm{kg}$ was performed via a Microsprayer
(PennCentury, Philadelphia, PA) as previously described [17]. All mice were sacrificed by deep anesthesia 2, 7, 14 or 21 days after the BLM instillation.

The TS group mice received $5 \mathrm{mg} / \mathrm{kg} /$ day of TS (Sigma-Aldrich, St. Louis, MO) in drinking water from 3 days before BLM challenge until sacrifice. Since a mouse drinks $500 \mathrm{~mL} / \mathrm{kg} /$ day of water in average, TS was dissolved at $10 \mu \mathrm{g} / \mathrm{mL}$ in the water. The mice with RGZ treatment received intraperitoneal injection of 10 $\mathrm{mg} / \mathrm{kg}$ of RGZ (Cayman Chemical, Ann Arbor, MI) daily from the day of BLM challenge until sacrifice. Each experimental group included 12 mice, 6 for bronchoalveolar lavage and 6 for lung pathology.

\subsection{Preparation and Analysis of Bronchoalveolar Lavage}

To evaluate inflammatory cell accumulation in the alveolar space, BAL fluid was collected by cannulating the trachea and lavaging the lung with three separate $1.0 \mathrm{~mL}$ volumes of sterile saline, each volume being instilled and withdrawn three times. The average volume retrieved was approximately $90 \%$, and the recovery rates did not differ between the groups. The fluid collections were combined and cooled to $4^{\circ} \mathrm{C}$. The lavage fluid was centrifuged at $300 \mathrm{~g}$ for $10 \mathrm{~min}$, and the cell count was determined on a fresh fluid specimen using a hemocytometer. The supernatants were stored at $-80^{\circ} \mathrm{C}$ until the measurement of mediators.

\subsection{Histopathological Determination of Lung Inflammation and Fibrosis}

The lungs were fixed by intratracheal instillation of $10 \%$ neutral phosphate-buffered formalin and paraffin embedded. The tissues were cut into $3-\mu \mathrm{m}$ sections and stained with hematoxylin-eosin (H-E) or Masson's trichrome for morphological analysis.

Fibrotic change was evaluated with Ashcroft score, a numerical fibrotic scoring scale, in histologic sections that were stained with Masson's trichrome [21]. A score of 0 - 1 was grouped as no fibrosis, 2 - 3 as minimal, 4 5 as moderate and $6-8$ as severe fibrosis. Grading was performed by a single investigator in a blinded fashion.

\subsection{Analysis of Collagen Content in the Lung}

Total lung collagen content was determined using the Sircol collagen assay (Biocolor Ltd., Belfast, United Kingdom) according to the manufacturer's instructions.

\subsection{Measurement of Cytokine and $\mathrm{PGF}_{2 \alpha}$ Levels in the Lung}

The levels of TGF- $\beta_{1}$, CCL2, and IL-6 in BAL fluid were measured using ELISA (R\&D Systems, Minneapolis, $\mathrm{MN}$ ) following the manufacturer's instruction. $\mathrm{PGF}_{2 \alpha}$ 
level was measured with EIA (Cayman Chemical, Ann Arbor, MI).

\subsection{Isolation of Mouse Lung Fibroblasts}

Lungs from untreated mice were digested for $45 \mathrm{~min}$ at $37^{\circ} \mathrm{C}$ in RPMI with $0.28 \mathrm{U} / \mathrm{mL}$ liberase blendzyme 3 and $60 \mathrm{U} / \mathrm{ml}$ DNase I, passed through a $70 \mu \mathrm{m}$ filter, centrifuged at $540 \times \mathrm{g}$ at $4^{\circ} \mathrm{C}$, and plated in tissue culture flasks in Dulbecco's modified Eagle's medium (DMEM) with $15 \%$ fetal bovine serum (FBS). Cells were passaged when subconfluent after harvest with trypsin-EDTA. Cells were used for experiments at passages 3 and 4 .

\subsection{Cell Proliferation Assay}

The effect of PPAR- $\gamma$ agonists on cell proliferation was assessed by an MTT assay. MLFs $\left(2 \times 10^{3} / 100 \mu \mathrm{L}\right)$ were seeded into the 96-well culture plates and incubated until they reached subconfluence. Thereafter, cells were washed with PBS, and original medium was replaced with medium containing no FBS. MLFs were cultured for another $24 \mathrm{~h}$ until treatment with $10 \%$ FBS in serum-free medium in the presence of RGZ or TS for 24 and 48 hours; MTT $(0.5 \mathrm{mg} / \mathrm{mL})$ was added in the last 3 hours. After the removal of the medium and the addition of DMSO to the flask, the absorbance at $570 \mathrm{~nm}$ was measured by use of a microplate reader in controls.

\subsection{Statistical Analysis}

Data are reported as mean \pm SEM. Differences among groups were determined using analysis of variance followed by post hoc analysis with the Bonferroni's test for multiple comparisons. A $\mathrm{p}$ value less than 0.05 was considered statistically significant.

\section{Results}

\subsection{Inflammatory Cell Accumulation in the Airspace after Bleomycin Administration}

To examine the effect of PPAR- $\gamma$ ligands on BLM-induced accumulation of inflammatory cells, we examined the number of inflammatory cells in BAL fluid obtained on days 2, 7, 14, and 21 (Figure 1). Intratracheal BLM significantly increased the cell count in BAL fluid on day 2, compared with the control mice that received PBS instillation $(p<0.05)$. Further accumulation of inflammatory cells occurred on days 7, 14, and 21. Either RGZ or TS significantly suppressed the BLM-induced cell accumulation on days 7,14 , and 21 as compared with the mice without a PPAR- $\gamma$ ligand $(\mathrm{p}<0.05)$.

\subsection{Lung Histopathology}

Since the most prominent accumulation of inflammatory

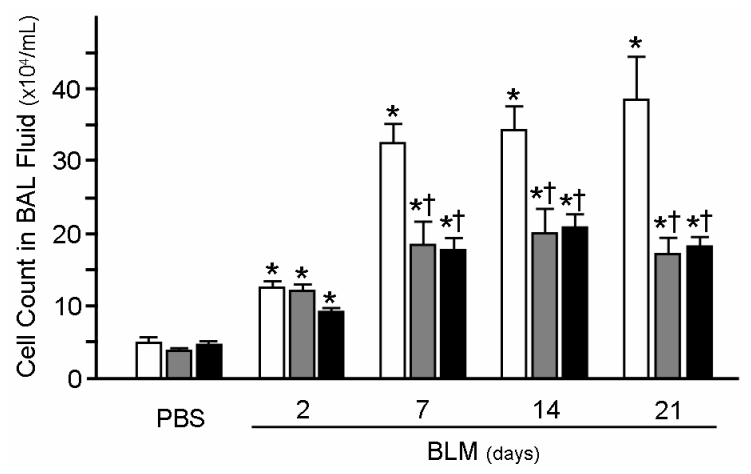

Figure 1. Cell counts in BAL fluid after the intratracheal instillation of PBS or BLM. The BLM challenge induced significant increases in the cell counts on day 2 and later compared with the control mice that received PBS. In the groups treated with TS (gray column) or RGZ (closed column), the BLM-induced increases in the cell counts were significantly attenuated compared with those without a PPAR- $\gamma$ agonist (open column). All values are expressed as the mean $\pm \mathrm{SE}(\mathrm{n}=6) .{ }^{*} \mathrm{p}<0.05$ was considered to be significantly different from the corresponding value of the control mice with PBS instillation. ${ }^{\dagger} \mathbf{p}<0.05$ was considered to be significantly different from the corresponding value of the mice that received BLM alone.

cells into the airspace was observed on day 7 and later, we evaluated lung pathology using the H-E stained samples obtained on day 7. Representative microscopic findings are shown in Figure 2. Compared with the control mice, the BLM administration induced marked hemorrhages and congestion with infiltration of inflammatory cells, which were ameliorated by the treatment with either RGZ or TS.

To examine the effect of PPAR- $\gamma$ agonists on BLMinduced fibrotic changes, lung pathology on day 21 were evaluated. Representative microscopic findings after Masson's trichrome staining are shown in Figure 3. In the animals without a PPAR- $\gamma$ agonist treatment, the BLM administration caused marked thickening of the alveolar septa and infiltration predominated by mononuclear cells with significant collagen expression. In the mice treated with TS or RGZ, these pathological changes induced by intratracheal BLM were significantly mitigated.

The Ashcroft sores for more quantitative assessment of the lung fibrotic changes were shown in Figure 4. On day 7 and later, the scores were significantly higher in the mice treated with BLM than in those with PBS ( $p<$ 0.05 ). On days 14 and 21, the treatment with either TS or RGZ significantly attenuated the BLM-induced increases in the score compared with the group without a PPAR- $\gamma$ agonist $(\mathrm{p}<0.05)$.

\subsection{Collagen Contents in the Lung}

The lungs harvested on day $2,7,14$, or 21 were analyzed 

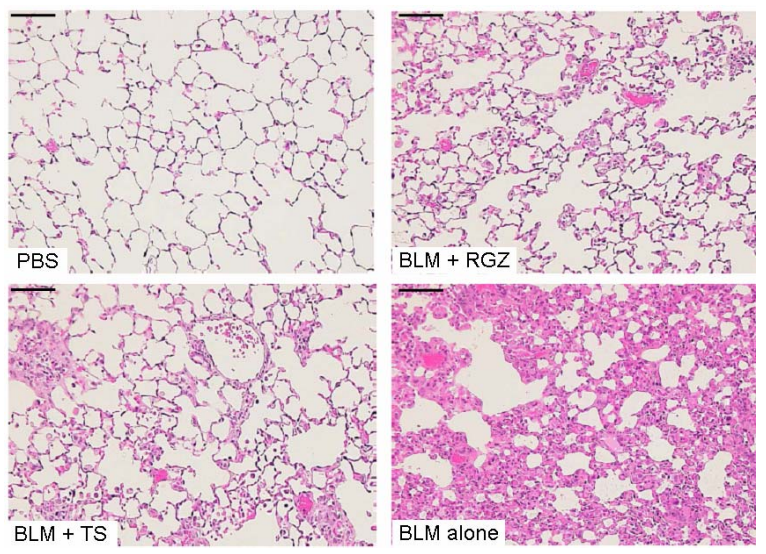

Figure 2. Representative examples of lung pathology 7 days after the instillation of PBS or BLM. Hematoxylin-eosin stain. Original magnification $\times 200$. Bars, $50 \mu \mathrm{m} . n=6$ in each group. Twenty fields were examined per section.
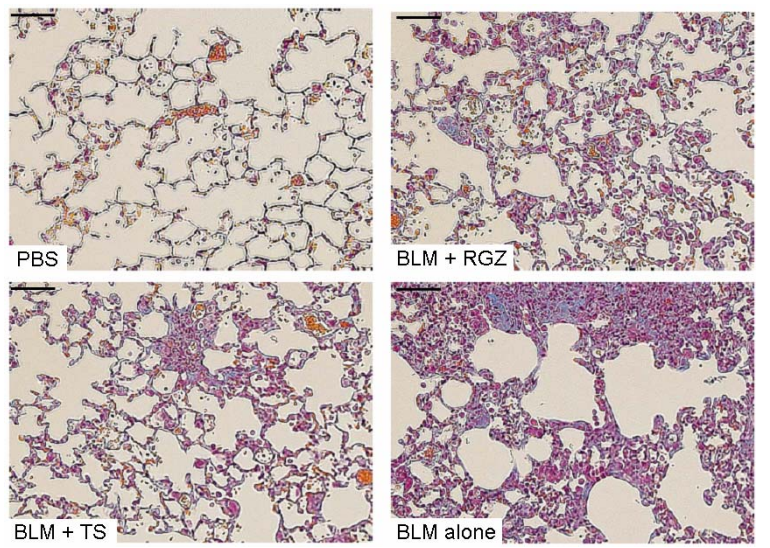

Figure 3. Representative examples of lung pathology 21 days after the instillation of PBS or BLM. Masson's trichrome stain. Original magnification $\times 200$. Bars, $50 \mu \mathrm{m}$. $n=6$ in each group. Twenty fields were examined per section.

for collagen content (Figure 5). In the mice without a PPAR- $\gamma$ agonist, intratracheal BLM caused significant increases in the collagen content of the lungs on days 14 and 21, compared with those administered with PBS $(\mathrm{p}<$ $0.05)$. On day 21 , the collagen content in the lungs was significantly less in the groups treated with TS or RGZ than in those without administration of a PPAR $-\gamma$ ligand $(\mathrm{p}<0.05)$.

\subsection{Levels of Profibrotic Cytokines in the Lung}

To validate the effect of PPAR- $\gamma$ agonists on BLM-induced upregulation of the cytokines associated with fibrosis, the levels of TGF- $\beta_{1}$, CCL2/MCP-1, and IL-6 were measured in BAL fluid with ELISA.

On days 7 and 14, the TGF- $\beta_{1}$ levels in the lung were significantly increased in the BLM-treated mice (Figure 6(a)). Whereas the increase in the TGF- $\beta_{1}$ level on days

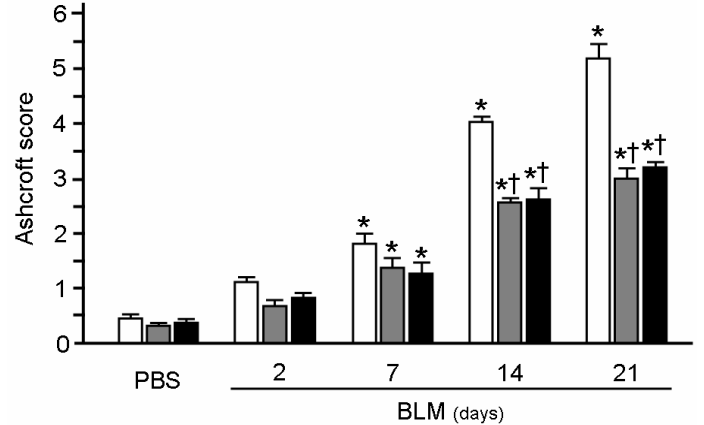

Figure 4. The Ashcroft sores for quantitative assessment of the lung fibrotic changes after the intratracheal instillation of PBS or BLM. In the BLM-treated mice, the scores on days 7,14 , and 21 were significantly higher than in the control mice with PBS instillation. In the groups treated with TS (gray column) or RGZ (closed column), the BLM-induced increases in the score were significantly suppressed on days 14 and 21 as compared with those without treatment with a PPAR- $\gamma$ agonist (open column). All values are expressed as the mean \pm SE $(n=6) . * p<0.05$ was considered to be significantly different from the corresponding value of the control mice with PBS instillation. ${ }^{\dagger} p<0.05$ was considered to be significantly different from the corresponding value of the mice administered BLM alone.

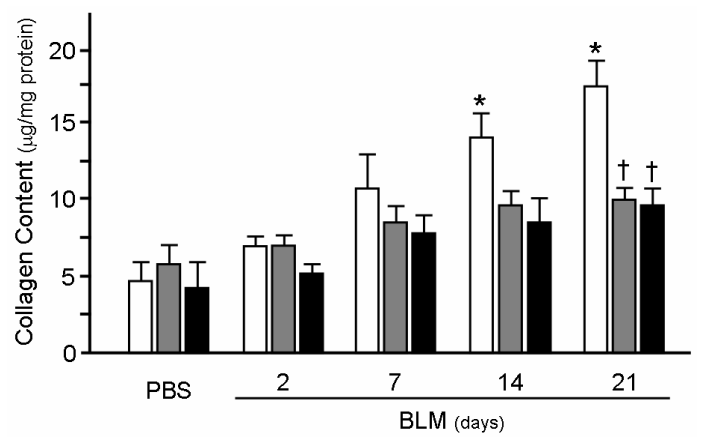

Figure 5. Collagen contents in the lung after the instillation of PBS or BLM. Intratracheal BLM caused significant increase in the collagen content in the lungs. In the groups treated with TS (gray column) or RGZ (closed column), the BLM-induced increase in the collagen content was significantly suppressed on day 21 as compared with those without a PPAR- $\gamma$ agonist (open column). All values are expressed as the mean $\pm \operatorname{SE}(n=6) .{ }^{*} p<0.05$ was considered to be significantly different from the corresponding value of the control mice with PBS instillation. ${ }^{\dagger} \mathbf{p}<0.05$ was considered to be significantly different from the corresponding value of the mice that received BLM alone.

7 and 14 was significantly suppressed by RGZ $(\mathrm{p}<0.05)$, there were no significant differences in the TGF- $\beta_{1}$ levels between the TS-treated mice and those without treatment with a PPAR- $\gamma$ agonist.

In the animals without a PPAR- $\gamma$ agonist, the levels of CCL2 in BAL fluid were significantly elevated 2 and 7 days after the BLM challenge (Figure 6(b)). In the RGZ-treated animals, the BLM-induced elevation of the 
CCL2 level was significantly suppressed on day 2 ( $p<$ 0.05 ). The levels of CCL2 were not different between the TS-treated mice and those without a PPAR- $\gamma$ agonist at any time point.

The BLM challenge significantly elevated the levels of IL-6 in the lung 2, 7, or 14 days after the intratracheal challenge (Figure 6(c)). Whereas the increases in the IL-6 levels on days 2, 7, and 14 were significantly suppressed by RGZ ( $p<0.05)$, there was no significant difference in the IL-6 levels between the TS-treated mice and those without administration of a PPAR- $\gamma$ agonist.

\subsection{PGF ${ }_{2 \alpha}$ Levels in the Lung}

Since the levels of the profibrotic cytokines were not changed by $\mathrm{TS}$, the level of $\mathrm{PGF}_{2 \alpha}$, a lipid mediator, was measured in BAL fluid with EIA (Figure 6(d)). The increases in the $\mathrm{PGF}_{2 \alpha}$ levels on days 2, 7, and 14 were significantly suppressed by TS $(\mathrm{p}<0.05)$, whereas there was no significant difference in the $\mathrm{PGF}_{2 \alpha}$ level between the RGZ-treated mice and those without treatment with a PPAR- $\gamma$ agonist.

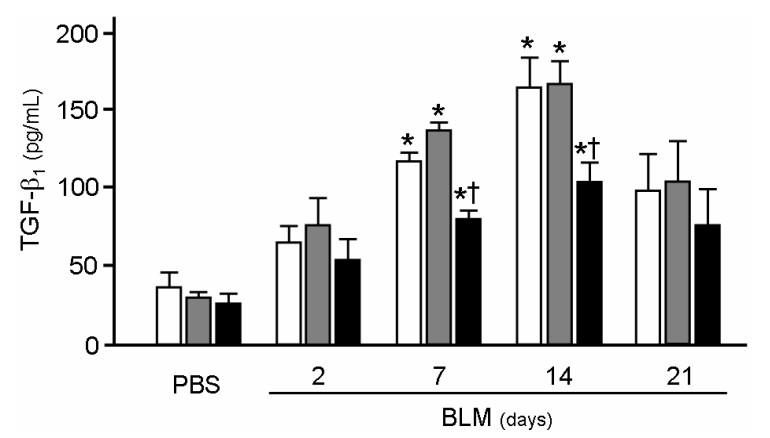

(a)

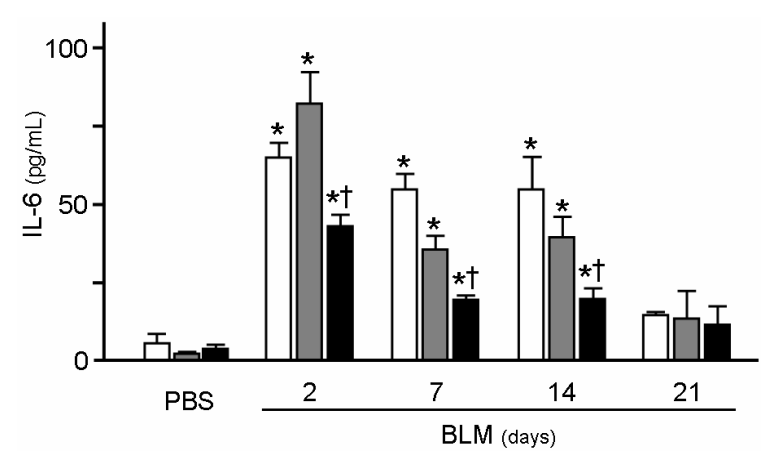

(c)

\subsection{Proliferation of Lung Fibroblast}

The number of MLFs was higher, by 1.51 - and 0.97 -fold with $10 \%$ FBS incubation after 24 and $48 \mathrm{~h}$, respectively, than in controls $(p<0.01)$ (Figure 7). At 24 h, 10 or 20 $\mu \mathrm{mol} / \mathrm{L}$ of TS significantly suppressed the proliferation of MLFs, which was induced by $10 \%$ FBS alone ( $p<$ 0.05) (Figure 7(a)). Although $5 \mu \mathrm{mol} / \mathrm{L}$ of RGZ made no change in the MLF number, 10 or $20 \mu \mathrm{mol} / \mathrm{L}$ of RGZ significantly decreased the cell number at $24 \mathrm{~h}$, compared with FBS alone $(p<0.05)$. At $48 \mathrm{~h}$, whereas $5 \mu \mathrm{mol} / \mathrm{L}$ of TS made no change in the proliferation of MLFs, 10 or $20 \mu \mathrm{mol} / \mathrm{L}$ of RGZ significantly reduced the number of MLFs, compared with FBS alone $(p<0.05)$ (Figure 7(b)). After co-incubation with FBS and 10 or $20 \mu \mathrm{mol} / \mathrm{L}$ of RGZ for $48 \mathrm{~h}$, the number of MLFs was lower than in the FBS alone $(\mathrm{p}<0.05)$.

\section{Discussion}

In the present study, it was found that two PPAR- $\gamma$ ligands, RGZ and TS, attenuated the BLM-induced inflammatory and fibrotic changes of the lung. The BLM-

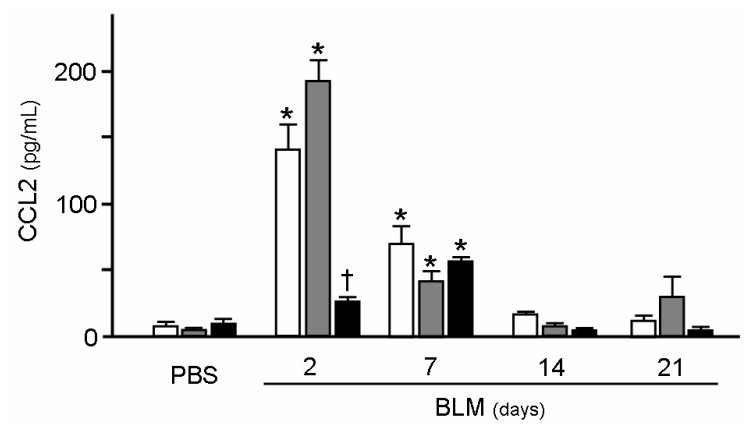

(b)

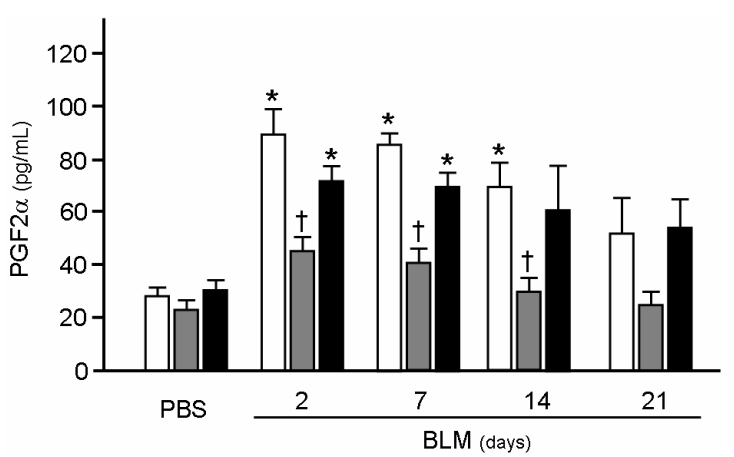

(d)

Figure 6. Levels of TGF- $\beta_{1}$, CCL2, IL-6, and PGF ${ }_{2 \alpha}$ in BAL fluid. (a) TGF- $\beta_{1}$ levels were elevated 7, 14, and 21 days after the BLM challenge, which was significantly attenuated by treatment with RGZ; (b) CCL2 level in the lung was elevated 2 and 7 days after the BLM challenge. The treatment with RGZ significantly suppressed the increase on day 2; (c) IL-6 levels were elevated 7, 14, and 21 days after the BLM challenge, which was significantly attenuated by the treatment with RGZ; (d) $\mathrm{PGF}_{2 \alpha}$ levels were elevated on days 2,7 , and 14 after the BLM challenge, which was significantly suppressed by treatment with TS. All values are expressed as the mean $\pm S E(n=6)$. Open, gray, and closed columns indicate mice without treatment with a PPAR- $\gamma$ agonist, those treated with TS, and those treated with RGZ, respectively. ${ }^{*} \mathbf{p}<0.05$ was considered to be significantly different from the corresponding value of the control mice with PBS instillation. ${ }^{\dagger} p<0.05$ was considered to be significantly different from the corresponding value of the mice administered BLM alone. 


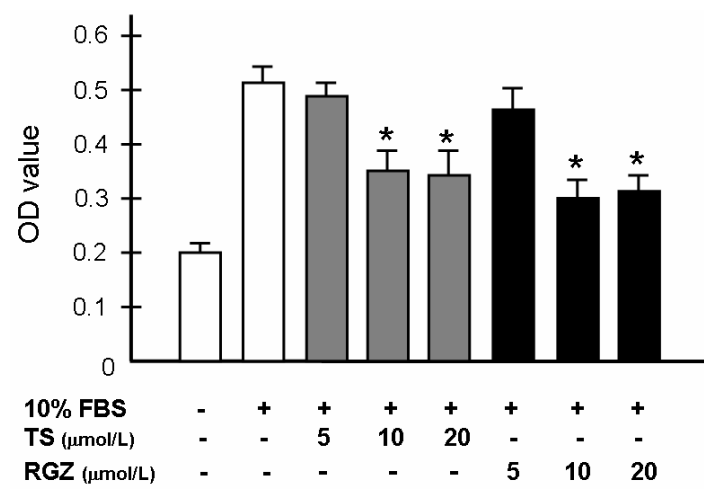

(a)

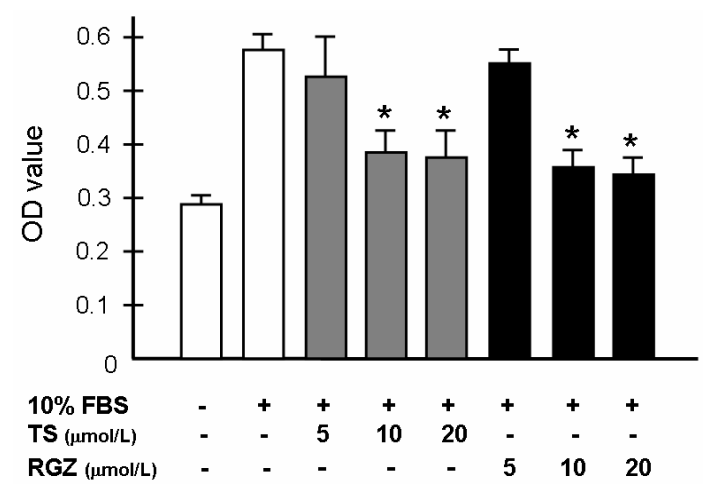

(b)

Figure 7. Effect of RSG and TS on proliferation of lung fibroblast. (a) At $24 \mathrm{~h}, 10$ or $20 \mu \mathrm{mol} / \mathrm{L}$ of TS or RGZ significantly suppressed proliferation of lung fibroblast; (b) After incubation with 10 or $20 \mu \mathrm{mol} / \mathrm{L}$ of TS or RGZ for 48 $h$, the number of murine lung fibroblast was lower than in the FBS alone. All values are expressed as the mean $\pm \mathrm{SE}$. ${ }^{*} \mathbf{p}<0.05$ was considered to be significantly different from the corresponding value of the FBS control.

induced increases in the levels of profibrotic cytokines TGF- $\beta_{1}$, CCL2, and IL- 6 were suppressed by the administration of RGZ. In the TS-treated mice, whereas the levels of these cytokines were not changed, the elevation of $\mathrm{PGF}_{2 \alpha}$, a lipid mediator associated with fibrosis, after the BLM challenge was attenuated. These results might indicate protective effects of PPAR- $\gamma$ ligands against the development of lung fibrosis through suppression of cytokines or a lipid mediator associated with fibrosis.

Among the cytokines and chemokines that have been implicated in the pathogenesis of BLM toxicity, particular relevance has been given to TGF- $\beta_{1}[16]$. In this study, we observed that the RGZ treatment attenuated the BLM-induced increase in TGF- $\beta_{1}$ in the lung, which is comparable with the results of previous studies $[22,23]$. Recently, Tan and coworkers showed that RGZ suppressed TGF- $\beta_{1}$-induced upregulation of E-cadherin, suggesting the inhibition of epithelial mesenchymal transition (EMT) by activation of PPAR- $\gamma$ [24]. TGF- $\beta_{1^{-}}$ induced EMT contributes to the de novo appearance of myofibroblasts in the lung. We considered that, in the animals treated with RGZ, the suppression of TGF- $\beta_{1}$ production might be critical for the attenuation of the BLM-induced lung fibrosis.

In addition to TGF- $\beta_{1}$, a relative contribution to lung fibrosis of members of the $\mathrm{CC}$ chemokines, such as monocyte chemotactic protein-1 (MCP-1/CCL2), has been proposed $[17,25]$. In this study, CCL2 was elevated in the lung 2 days after the BLM challenge, and it is significantly fallen by day 14 . CCL2, a chemokine responsible for mononuclear cell recruitment, is secreted by a variety of cell types such as lymphocytes, macrophages, fibroblasts, and endothelial cells [17]. We considered that, in the RGZ-treated mice, the decreased release of CCL2 might contribute not only to the attenuation of lung fibrosis but also to the suppression of inflammatory cell accumulation.

In a previous report, Genovese and colleagues demonstrated that administration of RGZ reduced mortality rate, accumulation of inflammatory cells in the alveolar compartment, edema formation, and histological evidence of BLM-induced lung injury [12]. Since our study was focused on the effect of PPAR- $\gamma$ agonists on lung fibrosis, the parameters of our study are different from those of theirs. Although the dose of BLM was similar, the survival rate was different between our study and theirs. In the study by Genovese and colleagues, $50 \%$ of the BLMtreated mice that had received vehicle died within 15 days [12]. In contrast, none of the animals died during the observation period in our study. We considered that this discrepancy might be due to the difference in the mouse strain and the way of drug administration. In our study, a Microsprayer was used so that the drug solution could be distributed homogenously in the lungs.

In the present study, we observed that the levels of profibrotic cytokines in BAL fluid were not affected by TS. It was previously reported that treatment of cultured alveolar epithelial cells with TS reduces TGF- $\beta_{1}$-induced collagen I production and cell migration [26]. We therefore hypothesized that the inhibitory effect of TS might be mediated by other profibrotic mediator and examined the levels of $\mathrm{PGF}_{2 \alpha}$ in the lungs. $\mathrm{PGF}_{2 \alpha}$, is a lipid mediator, which has been shown to promote BLM-induced lung fibrosis independently of TGF- $\beta_{1}[20]$. We found that the treatment with TS significantly suppressed the BLM-induced increase in $\mathrm{PGF}_{2 \alpha}$. Oga and colleagues reported that $\mathrm{PGF}_{2 \alpha}$ is abundant in BAL fluid of patients with idiopathic pulmonary fibrosis and stimulates proliferation and collagen production of lung fibroblasts [20]. We speculated that the inhibitory effect of TS on the BLM-induced fibrosis might be mediated by suppression of $\mathrm{PGF}_{2 \alpha}$ rather than profibrotic cytokines such as TGF$\beta_{1}$.

TS was originally developed as an ARB. Although 
some ARBs have PPAR- $\gamma$ agonist activity, the agonistic PPAR- $\gamma$ effect of TS is known to be greater than that of other ARBs [27]. Benson and coworkers tested several ARBs for their capacity to activate the expression of PPAR $-\gamma$ target genes and found that, at physiological condition, only TS induced a substantial response [27]. On the other hand, renin-angiotensin system is associated with pathogenesis of experimental lung fibrosis. It was reported that intratracheal BLM induced overexpression of angiotensin II type 1 receptor in inflammatory immune cells, alveolar type II cells, and fibroblasts [15]. In addition, administration of an angiotensin-converting enzyme inhibitor ramipril reduced collagen deposition after BLM challenge [28]. It remains to be determined whether the antifibrotic effect of TS is through activation of PPAR- $\gamma$ pathway or blockade of renin-angiotensin system.

In this study, FBS-induced proliferation of MLFs was attenuated by higher concentrations of RGZ and TS. The antiproliferative effects of PPAR- $\gamma$ agonists have been demonstrated in some cell types. Benson and coworkers showed that TS inhibited proliferation of cardiac fibroblast in a dose-dependent fashion [29]. In addition, Lin and colleagues reported that RGZ treatment inhibits FBS-induced proliferation of cultured human lung fibroblast [30]. We considered that antiproliferative effects of PPAR- $\gamma$ agonists on lung fibroblast might be associated with the attenuation of BLM-induced fibrosis.

The molecular mechanisms underlying the antifibrotic effects of PPAR $-\gamma$ are the subject of intense investigation. In fibroblasts, ligand-activated PPAR $-\gamma$ blocks profibrotic signaling triggered by TGF- $\beta_{1}$ and Wnt- $\beta$-catenin and interferes with downstream signal transduction [31, 32]. In this study, we observed reduction of TGF- $\beta_{1}$ levels in the lung by RGZ as well as suppression of $\mathrm{PGF}_{2 \alpha}$ by TS. Xu and coworkers showed that stimulation of the human FP prostanoid receptor with $\mathrm{PGF}_{2 \alpha}$ induces sequential activation of Ras and Raf kinases, followed by Tcf transcriptional activation [33]. Since Tcf forms a complex with $\beta$-catenin to activate transcription of Wnt, we speculated that TS-induced suppression of $\mathrm{PGF}_{2 \alpha}$ might be a key mechanism of its protective effect. The effect of PPAR- $\gamma$ ligands on these signaling cascades should be the subject of further investigation.

In conclusion, two PPAR- $\gamma$ ligands, RGZ and TS, exert protective effects on BLM-induced lung fibrosis possibly through suppression of different profibrotic mediators. RGZ suppressed the BLM-induced increases in profibrotic cytokines, TGF- $\beta$, MCP-1, and IL-6, whereas TS mitigated the increases in $\mathrm{PGF}_{2 \alpha}$ in the lung. Although its efficacy remains to be evaluated in a more clinically relevant model, a PPAR- $\gamma$ modulator could be considered as a candidate of a therapeutic modality for noninfectious lung injury and subsequent fibrotic changes.

\section{Acknowledgements}

The authors thank Dr. Tomomi Ueda and Ms. Miyuki Yamamoto for their excellent technical assistance.

\section{REFERENCES}

[1] American Thoracic Society, "Idiopathic Pulmonary Fibrosis: Diagnosis and Treatment. International Consensus Statement. American Thoracic Society (ATS), and the European Respiratory Society (ERS)," American Journal of Respiratory and Critical Care Medicine, Vol. 161, No. 2, 2000, pp. 646-664. doi:10.1164/ajrccm.161.2.ats3-00

[2] A. Moeller, K. Ask, D. Warburton, et al., "The Bleomycin Animal Model: A Useful Tool to Investigate Treatment Options for Idiopathic Pulmonary Fibrosis?" International Journal of Biochemistry and Cell Biology, Vol. 40, No. 3, 2008, pp. 362-382. doi:10.1016/j.biocel.2007.08.011

[3] D. H. Bowden, "Unraveling Pulmonary Fibrosis: The Bleomycin Model," Laboratory Investigation, Vol. 50, No. 5, 1984, pp. 487-488.

[4] C. Agostini and C. Gurrieri, "Chemokine/Cytokine Cocktail in Idiopathic Pulmonary Fibrosis," Proceedings of the American Thoracic Society, Vol. 3, No. 4, 2006, pp. $357-$ 363. doi:10.1513/pats.200601-010TK

[5] R. L. Riha, I. A. Yang, G. C. Rabnott, et al., "Cytokine Gene Polymorphisms in Idiopathic Pulmonary Fibrosis," Internal Medicine Journal, Vol. 34, No. 3, 2004, pp. 126129. doi:10.1111/j.1444-0903.2004.00503.x

[6] Y. Wang, J. Santos, R. Sakurai, et al., "Peroxisome Proliferator-Activated Receptor Gamma Agonists Enhance Lung Maturation in a Neonatal Rat Model," Pediatric Research, Vol. 65, No. 2, 2009, pp. 150-155. doi:10.1203/PDR.0b013e3181938c40

[7] T. J. Standiford, V. G. Keshamouni and R. C. Reddy, "Peroxisome Proliferator-Activated Receptor- $\gamma$ as a Regulator of Lung Inflammation and Repair," Proceedings of the American Thoracic Society, Vol. 2, No. 3, 2005, pp. 226-231. doi:10.1513/pats.200501-010AC

[8] M. Ricote, A. C. Li, T. M. Willson, et al., "The Peroxisome Proliferator-Activated Receptor-Gamma Is a Negative Regulator of Macrophage Activation," Nature, Vol. 391, No. 6662, 1998, pp. 79-82. doi:10.1038/34178

[9] K. Asada, S. Sasaki, T. Suda, et al., "Antiinflammatory Roles of Peroxisome Proliferators-Activated Receptor Gamma in Human Alveolar Macrophages," American Journal of Respiratory and Critical Care Medicine, Vol. 169, No. 2, 2004, pp. 195-200. doi:10.1164/rccm.200207-7400C

[10] R. A. Daynes and D. C. Jones, "Emerging Roles of PPARs in Inflammation and Immunity," Nature Reviews Immunology, Vol. 2, No. 10, 2002, pp. 748-759. doi:10.1038/nri912

[11] J. M. Lehmann, L. B. Moore, T. A. Smith-Oliver, et al., "An Antidiabetic Thiazolidinedione Is a High Affinity Ligand for Peroxisome Proliferator-Activated Receptor $\gamma$ (PPAR- $\gamma$ )," Journal of Biological Chemistry, Vol. 270, 
No. 22, 1995, pp. 12953-12956. doi: $10.1074 / \mathrm{jbc} .270 .22 .12953$

[12] T. Genovese, S. Cuzzocrea, R. Di Paola, et al., "Effect of Rosiglitazone and 15-Deoxy- $\Delta^{12,14}$-Prostaglandin $\mathrm{J}_{2}$ on Bleomycin-Induced Lung Injury," European Respiratory Journal, Vol. 25, No. 2, 2005, pp. 225-234. doi:10.1183/09031936.05.00049704

[13] T. Tagami, H. Yamamoto, K. Moriyama, et al., “A Selective Peroxisome Proliferator-Activated Receptor-Gamma Modulator, Telmisartan, Binds to the Receptor in a Different Fashion from Thiazolidinediones," Endocrinology, Vol. 150, No. 2, 2009, pp. 862-870. doi:10.1210/en.2008-0502

[14] M. Schupp, J. Janke, R. Clasen, et al., “Angiotensin Type 1 Receptor Blockers Induce Peroxisome Proliferator-Activated Receptor-Gamma Activity," Circulation, Vol. 109, No. 17, 2004, pp. 2054-2057. doi:10.1161/01.CIR.0000127955.36250.65

[15] M. Otsuka, H. Takahashi, M. Shiratori, et al., "Reduction of Bleomycin Induced Lung Fibrosis by Candesartan Cilexetil, an Angiotensin II Type 1 Receptor Antagonist," Thorax, Vol. 59, No. 1, 2004, pp. 31-38. doi:10.1136/thx.2003.000893

[16] T. J. Broekelmann, A. H. Limper, T. V. Colby, et al., "Transforming Growth Factor $\beta_{1}$ is Present at Sites of Extracellular Matrix Gene Expression in Human Pulmonary Fibrosis," Proceedings of the National Academy of Sciences of the United States of America, Vol. 88, No. 15, 1991, pp. 6642-6646. doi:10.1073/pnas.88.15.6642

[17] R. E. Smith, R. M. Strieter, K. Zhang, et al., "A Role for C-C Chemokines in Fibrotic Lung Disease," Journal of Leukocyte Biology, Vol. 57, No. 5, 1995, pp. 782-787.

[18] I. Shahar, E. Fireman, M. Topilsky, et al., "Effect of IL-6 on Alveolar Fibroblast Proliferation in Interstitial Lung Diseases," Clinical Immunology and Immunopathology, Vol. 79, No. 3, 1996, pp. 244-251. doi:10.1006/clin.1996.0075

[19] F. Saito, S. Tasaka, K. Inoue, et al., "Role of Interleukin6 in Bleomycin-Induced Lung Inflammatory Changes in Mice," American Journal of Respiratory Cell and Molecular Biology, Vol. 38, No. 5, 2008, pp. 566-571. doi:10.1165/rcmb.2007-02990C

[20] T. Oga, T. Matsuoka, C. Yao, et al., "Prostaglandin $\mathrm{F}_{2 \alpha}$ Receptor Signaling Facilitates Bleomycin-Induced Pulmonary Fibrosis Independently of Transforming Growth Factor- $\beta$," Nature Medicine, Vol. 15, No. 12, 2009, pp. 1426-1430. doi:10.1038/nm.2066

[21] T. Ashcroft, J. M. Simpson and V. Timbrell, "Simple Method of Estimating Severity of Pulmonary Fibrosis on a Numerical Scale," Journal of Clinical Pathology, Vol. 41, No. 4, 1988, pp. 467-470. doi:10.1136/jcp.41.4.467

[22] K. Zhang, K. C. Flanders and S. H. Phan, "Cellular Localization of Transforming Growth Factor-Beta Expression in Bleomycin-Induced Pulmonary Fibrosis," American Journal of Pathology, Vol. 147, No. 2, 1995, pp. 352361.

[23] N. Khalil, T. V. Parekh, R. N. O'Connor, et al., "Differ- ential Expression of Transforming Growth Factor-Beta Type I and II Receptors by Pulmonary Cells in Bleomycin-Induced Lung Injury: Correlation with Repair and Fibrosis," Expimental Lung Research, Vol. 28, No. 3, 2002 , pp. 233-250. doi:10.1080/019021402753570527

[24] X. Tan, H. Dagher, C. A. Hutton, et al., "Effects of PPAR Gamma Ligands on TGF- $\beta_{1}$-Induced Epithelial-Mesenchymal Transition in Alveolar Epithelial Cells," Respiratory Research, Vol. 11, 2010, p. 21. doi:10.1186/1465-9921-11-21

[25] K. Zhang, M. Gharaee-Kermani, M. L. Jones, et al., "Lung monocyte Chemoattractant Protein-1 Gene Expression in Bleomycin-Induced Pulmonary Fibrosis," Journal of Immunology, Vol. 153, No. 10, 1994, pp. 4733-4741.

[26] S. T. Buckley, C. Medina and C. Ehrhardt, "Differential Susceptibility to Epithelial-Mesenchymal Transition (EMT) of Alveolar, Bronchial and Intestinal Epithelial Cells in Vitro and the Effect of Angiotensin II Receptor Inhibition," Cell and Tissue Research, Vol. 342, No. 1, 2010, pp. 39-51. doi:10.1007/s00441-010-1029-x

[27] S. C. Benson, H. A. Pershadsingh, C. I. Ho, et al., "Identification of Telmisartan as a Unique Angiotensin Ii Receptor Antagonist with Selective PPAR Gamma-Modulating Activity," Hypertension, Vol. 43, No. 5, 2004, pp. 993-1002. doi:10.1161/01.HYP.0000123072.34629.57

[28] R. P. Marshall, P. Gohlke, R. C. Chambers, et al., "Angiotensin II and the Fibroproliferative Response to Acute Lung Injury," American Journal of Physiology Lung Cellular and Molecular Physiology, Vol. 286, No. 1, 2004, pp. L156-L164. doi:10.1152/ajplung.00313.2002

[29] S. C. Benson, R. Iguchi, C. I. Ho, et al., "Inhibition of Cardiovascular Cell Proliferation by Angiotensin Receptor Blockers: Are All Molecules the Same?" Journal of Hypertension, Vol. 26, No. 5, 2008, pp. 973-980. doi:10.1097/HJH.0b013e3282f56ba5

[30] Q. Lin, L. P. Fang, W. W. Zhou, et al., "Rosiglitazone Inhibits Migration, Proliferation, and Phenotypic Differentiation in Cultured Human Lung Fibroblasts," Experimental Lung Research, Vol. 36, No. 2, 2010, pp. 120-128. doi:10.3109/01902140903214659

[31] A. K. Ghosh, S. Bhattacharyya, G. Lakos, et al., "Disruption of Transforming Growth Factor $\beta$ Signaling and Profibrotic Responses in Normal Skin Fibroblasts by Peroxisome Proliferator-Activated Receptor $\gamma$," Arthritis and Rheumatism, Vol. 50, No. 4, 2004, pp. 1305-1318. doi:10.1002/art.20104

[32] D. Lu and D. A. Carson, "Repression of $\beta$-Catenin Signaling by PPAR $\gamma$ Ligands," European Journal of Pharmacology, Vol. 636, No. 1-3, 2010, pp. 198-202. doi:10.1016/j.ejphar.2010.03.010

[33] W. Xu, C. L. Chou, D. D. Israel, et al., "PGF ${ }_{2 \alpha}$ Stimulates FP Prostanoid Receptor Mediated Crosstalk between Ras/Raf Signaling and Tcf Transcriptional Activation," Biochemical and Biophysical Research Communication, Vol. 381, No. 4, 2009, pp. 625-629. doi:10.1016/j.bbrc.2009.02.102 